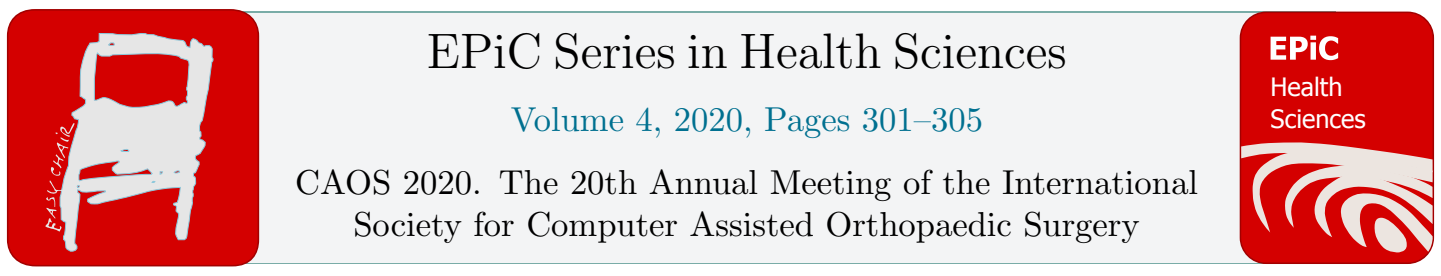

\title{
Using a robotics path planning algorithm to assess the risk of mobile bearing dislocation in lateral unicompartmental knee replacement.
}

\author{
Irene Yang ${ }^{1 \dagger}$, Jonathan D. Gammell ${ }^{2 *}$, David W. Murray ${ }^{1 \dagger}$ and Stephen J. \\ Mellon $^{1 \dagger}$ \\ ${ }^{1}$ Oxford Orthopaedic Engineering Centre, NDORMS, University of Oxford, UK. \\ ${ }^{2}$ Oxford Robotics Institute, Department of Engineering Science, University of Oxford, UK. \\ irene.yang@ndorms.ox.ac.uk
}

\begin{abstract}
Dislocation of the bearing occurs in 1 - $6 \%$ of Oxford Domed Lateral (ODL) mobile bearing unicompartmental knee replacements (UKRs). Dislocations occur in flexion as the lateral ligaments are lax in this position allowing the knee to distract. Anterior and posterior dislocations are rare: clinically, their dislocation rates are acceptable. Most dislocations tend to occur medially, with the bearing sitting on top of the tibial wall. Using robotics path planning algorithms and a modified Open Motion Planning Library (OMPL) Graphical User Interface (GUI), a dislocation analysis tool was developed to assess the minimum amount of vertical distraction of the femoral component relative to the tibial component required for the mobile bearing to dislocate. In the tool, the Rapidlyexploring Random Trees (RRT) algorithm was applied to the mobile bearing, which enabled autonomous movement of the bearing from a non-dislocated to a dislocated position. Testing increased the relative distance between the femoral component and the tibial component: vertically $(2-6 \mathrm{~mm})$ and mediolaterally $(0-4 \mathrm{~mm})$ in $0.25 \mathrm{~mm}$ increments resulting in a total of 289 configurations. For each configuration, the tool assessed whether mobile bearing dislocation was possible (either medially, laterally, anteriorly or posteriorly). For each mediolateral translation distance, the minimum vertical distraction required for dislocation was recorded. To validate the tool, dislocation results were compared to measurements taken using a custom-built mechanical rig. The minimum amount of distraction required for medial dislocation was similar for the dislocation analysis tool ( 3.75 to $4.75 \mathrm{~mm}$ ) as compared to a custom-built mechanical rig ( 2.5 to $4 \mathrm{~mm}$ ). The amount of distraction for a medial dislocation was much smaller than that for an anterior or posterior dislocation $(6$ to $6.25 \mathrm{~mm}$ ). This explains why medial dislocations are more common. Future work will use this tool to inform implant design,
\end{abstract}

\footnotetext{
* Designed the study, analyzed and interpreted the data, wrote and revised the manuscript.

Conceived and designed the study, analyzed and interpreted the data, wrote and revised the manuscript.
} 
Using a robotics path planning algorithm to assess the risk of mobile bearing dislocation... Yang et al.

with the aim to reduce the risk of medial dislocation to match that of anterior/posterior dislocation, which is clinically acceptable.

\section{Introduction}

The Oxford Domed Lateral (ODL) implant was introduced to restore the normal anatomy and kinematics of the knee. Although the clinical results are good, 1-6\% of mobile bearings dislocate[1,2]. Dislocations tend to occur in flexion as in this position, the lateral ligaments are lax, enabling lateral compartment distraction[3]. Whilst anterior and posterior dislocations are possible, the dislocation rate in these directions are clinically acceptable. Anterior and posterior dislocations are rare: clinically, their dislocation rates are acceptable. Most dislocations tend to occur medially, with the bearing sitting on top of the tibial wall.

The Open Motion Planning Library (OMPL) (Houston, Texas, USA) is an open-source path planning library consisting of various state-of-the-art sampling-based motion planning algorithms[4]. In robotics, path planning algorithms are frequently used to solve challenging problems encountered in autonomous motion. Path planning algorithms identify of a series of steps connecting the robot's start state to some goal state while avoiding collisions with the environment. Rapidly-exploring Random Trees (RRT) [5] is a widely used path planning algorithm based on random sampling of robot states throughout the planning space.

This study reports the preliminary results using a robotics path planning algorithm, RRT, and a modified OMPL Graphical User Interface (GUI), to construct a dislocation analysis tool to assess the minimum amount of vertical distraction of the femoral component relative to the tibial component required for mobile bearing dislocation to occur. A convergence test was carried out to identify the optimal search time and number of search iterations for RRT and the final dislocation results were validated against data obtained using a custom-built mechanical rig.

\section{Methodology}

For all testing, the starting position was such that the top of the dome of the tibial component, was aligned with the bottom of the spherical femoral component.

For the robotics dislocation analysis tool, Computer Aided Design (CAD) models of the ODL components (small femur, $\mathrm{C}$ tibia, and 3 bearing) were obtained from Zimmer Biomet (Swindon, UK). For the simulation, the OMPL GUI[4] was modified to receive the ODL components: the femoral and tibial components defined the "environment" and the mobile bearing defined the "robot". The relative distance between the femoral and tibial components was increased: vertically $(2-6 \mathrm{~mm})$ and mediolaterally $(0-4 \mathrm{~mm})$ in $0.25 \mathrm{~mm}$ increments ( 289 configurations). For each configuration, the RRT algorithm was applied to the mobile bearing to assess whether dislocation was possible, and to determine the minimum Vertical Distraction to Dislocation (VDtD). RRT parameters (allowable assessment time \& no. of attempts) were optimised via a convergence test. Path step length was set to $1.25 \mathrm{~mm}$ and collision resolution to $0.0001 \mathrm{~mm}$. A bounding box was used to constrain the search area. The box was defined by the anterior, posterior and lateral edges of the tibial component, within which the centre of mass of the bearing had to fall. Medially, the bounding box extended a full bearing width. Medial dislocation (Figure 1) occurred when the mobile bearing entered the "goal area" defined anteroposteriorly by the anterior and posterior edges of the tibial component, medially by the midpoint of the tibial wall, and laterally by the midpoint between the highest point of the domed surface and the medial tibial wall. 
Using a robotics path planning algorithm to assess the risk of mobile bearing dislocation... Yang et al.

For testing using the mechanical rig, assessment was completed with a modified version of the rig described by Weston-Simons[6]. From the starting point, testing increased the relative distance between the femoral and tibial components: vertically $(0-8 \mathrm{~mm})$ and mediolaterally (ML) $(0-4 \mathrm{~mm})$ in $0.25 \mathrm{~mm}$ increments (561 configurations). For each configuration, the bearing was manipulated to assess whether dislocation was possible, and to determine the VDtD.

\section{Results}

The dislocation analysis tool was successfully modified to receive the ODL implant components. Convergence testing identified that at least 145 seconds and 25 iterations are required for maximum solution identification. However, within 75 seconds and 10 iterations, 95\% of the solutions had been identified.

The VDtD was assessed using the dislocation analysis tool was compared with that obtained using a mechanical rig (Figure 1, Table 1). As ML translation increases from $0 \mathrm{~mm}$ to $4 \mathrm{~mm}$, the VDtD medially of the mobile bearing reduces. For the mechanical rig, the reduction is from $4 \mathrm{~mm}$ to $2.5 \mathrm{~mm}$, and for the dislocation analysis tool, the reduction is from $4.75 \mathrm{~mm}$ to $3.75 \mathrm{~mm}$.
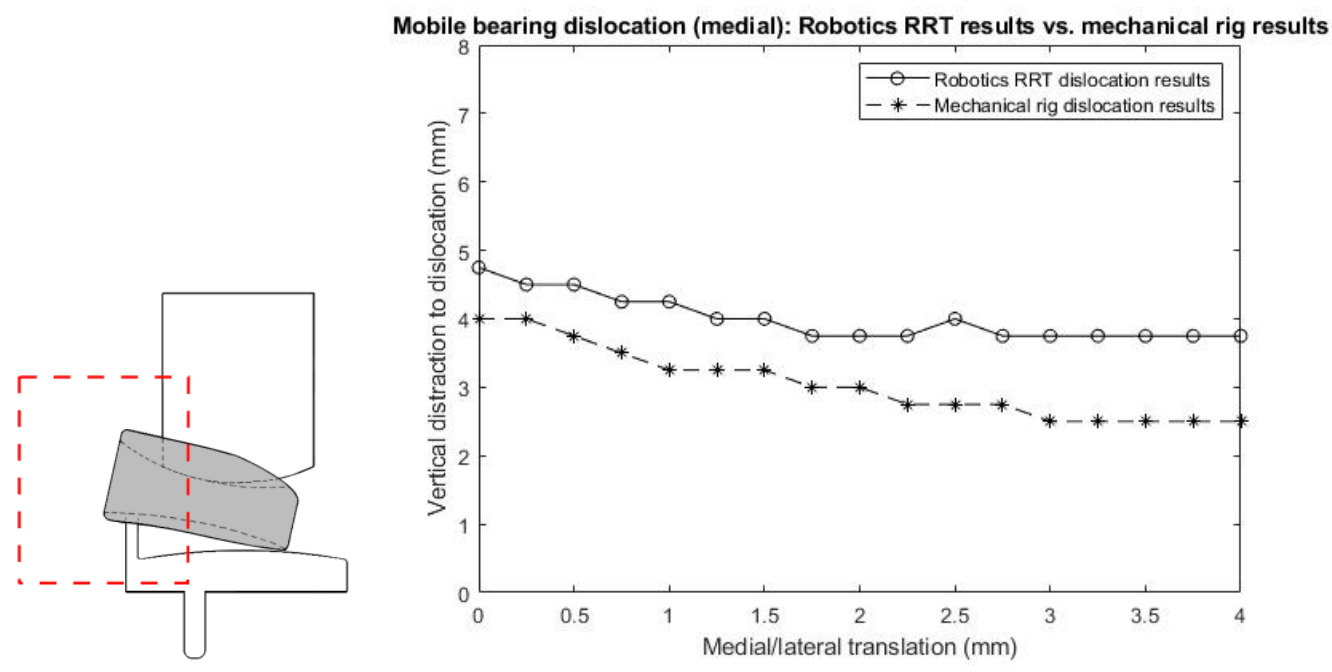

Figure 1: Medial dislocation of the mobile bearing (grey) (left) with goal area (red). Medial dislocation of the mobile bearing: dislocation analysis tool vs. mechanical rig. 
Using a robotics path planning algorithm to assess the risk of mobile bearing dislocation... Yang et al.

\begin{tabular}{ccccc}
\hline & \multicolumn{4}{c}{ Vertical Distraction to Dislocation (mm) } \\
ML translation (mm) & Medially & Laterally & Anteriorly & Posteriorly \\
\hline 0 & 4 & 2.75 & 6 & 6.25 \\
4 & 2.5 & 2.75 & 6 & 6.25 \\
\multicolumn{4}{c}{ Vertical Distraction to Dislocation (medially only) } \\
& \multicolumn{4}{c}{$(\mathbf{m m})$} \\
\hline ML translation (mm) & Mechanical rig & Robotics tool \\
\hline \multicolumn{4}{c}{4} & 4.75 \\
4 & 2.5 & 3.75 \\
Table 1: Top: Medial, lateral, anterior and posterior dislocation results obtained using a mechanical rig.
\end{tabular}
Bottom: Comparison of medial dislocation results obtained using the dislocation analysis tool vs. the mechanical rig.

Compared with mechanical rig results, the dislocation analysis tool under-predicts the VDtD medially (mean difference $0.96 \pm 0.25 \mathrm{~mm}$ ).

With the mechanical rig, the VDtD anteriorly $(6 \mathrm{~mm})$ and posteriorly $(6.25 \mathrm{~mm})$ were independent of ML position.

\section{Discussion \& Conclusion}

This study demonstrates a novel and successful application of a robotics path planning algorithm to the clinical problem of mobile bearing dislocation. As the laxity of the knee (and thus the amount of distraction) varies between patients, the risk of dislocation was assessed by the minimum amount of distraction that would allow a dislocation to occur.

The dislocation analysis tool was validated by comparing its results to the mechanical rig results. The robotics tool under-predicted the VDtD medially by $0.96 \pm 0.25 \mathrm{~mm}$. However, with altering ML translation, the trends were the same suggesting the analysis tool is valid. The discrepancy between the results may arise either from the mechanical rig, or from the robotics tool. With the mechanical rig, experimental error is likely since we used 3D printed parts (printing error compared with the CAD, warping of the 3D printed plastic components following extended use). To assess how much this contributes to the discrepancy seen in the results, we hope to quantify the 3D printing error in future. Further the rig is prone to bias (manually applied dislocation force, subjective dislocation judgement, interpolation bias during testing). By introducing the robotics tool, we have addressed many of the shortfalls with the rig. Whilst the robotics tool eliminated the user, and its associated possible errors, the tool is a probabilistic tool, dependent on optimising the search parameters, such as search time, number of searches and the path step length. Optimising the tool may improve the chances of the tool finding dislocations should they exist, and also reduce the chances of finding false negatives, which is an inherent problem of RRT. Despite these possible errors, the robotics tool shows excellent agreement with the results obtained using the mechanical rig. We plan to conduct further work on this tool, with the aim to reduce the discrepancy between the results obtained using the mechanical rig or the robotics tool to below $1 \mathrm{~mm}$. Finally, greater consideration is required with respect to the defined starting point for testing, to optimise the clinical applicability of the results.

Treating the tool as a comparative tool, the VDtD medially (2.5 to $4 \mathrm{~mm}$ ) was much less than that for an anterior or posterior dislocation $(6$ or $6.25 \mathrm{~mm})$. This explains why medial dislocation is more likely to occur than anterior or posterior dislocation. The amount of distraction required for lateral 
Using a robotics path planning algorithm to assess the risk of mobile bearing dislocation... Yang et al.

dislocation was less than that required for a medial dislocation. However, when the lateral compartment is distracted the lateral ligament and other soft tissues are tight[3], preventing a lateral dislocation.

Anterior and posterior bearing dislocation rarely occur and this dislocation rate is acceptable. Tokuhara et al. [7] studied the laxity of the lateral side of the knee in flexion and showed that the lateral compartment distracts on average $6.7 \mathrm{~mm}$. This is similar to the amount required for an anterior or posterior dislocation, probably explaining why these are rare. If the design of the implant could be modified so the amount of distraction for a medial dislocation was similar to that of an anterior or posterior dislocation, then dislocation risk would drop to an acceptable level. We plan to use the dislocation analysis tool to assess potential new designs that increase the VDtD medially with the aim to reduce the risk of medial dislocations to an acceptable level.

\section{Acknowledgements}

IY would like to thank the Clarendon Fund, Oxford University Press at University of Oxford for funding her DPhil studies.

\section{References}

1. Walker, T., et al., Mid-term results of lateral unicondylar mobile bearing knee arthroplasty: a multicentre study of 363 cases. The bone \& joint journal, 2018. 100-B(1): p. 42-49.

2. Pandit, H., et al., Mobile bearing dislocation in lateral unicompartmental knee replacement. The Knee, 2010. 17(6): p. 392-7.

3. Meister, B.R., et al., Anatomy and Kinematics of the Lateral Collateral Ligament of the Knee. The American Journal of Sports Medicine, 2000. 28(6): p. 869-878.

4. S Sucan, I.A., M. Moll, and L.E. Kavraki, The Open Motion Planning Library. IEEE Robotics \& Automation Magazine, 2012. 19(4): p. 72 - 82.

5. $\quad$ LaValle, S.M., Rapidly-exploring Random Trees: A New Tool for Path Planning. 1998.

6. Weston-Simons, J.S., et al., An analysis of dislocation of the domed Oxford Lateral Unicompartmental Knee Replacement. The Knee, 2014. 21(1): p. 304-9.

7. Tokuhara, Y., et al., The flexion gap in normal knees - an MRI study. J Bone Joint Surg [Br], 2004. 86B1: p. 1133-6. 